OPEN

SUBJECT AREAS:

DIFFERENTIATION

ANIMAL DISEASE MODELS

KIDNEY

CELL PROLIFERATION

Received

25 March 2013

Accepted

9 September 2013

Published

8 October 2013

Correspondence and requests for materials should be addressed to

N.O.L. (nlitbarg@ gmail.com)

\section{A Novel Model of Surgical Injury in Adult Rat Kidney: A "Pouch Model"}

\author{
Natalia O. Litbarg ${ }^{1,2}$, Snezana Vujicici, ${ }^{1,}$, Suman Setty ${ }^{1,2}$, Periannan Sethupathi ${ }^{3,4}$, George Dunea $^{3}$, \\ Jose A. Arruda' \& Ashok K. Singh ${ }^{3}$ \\ 'University of Illinois at Chicago, Department of Medicine, Section of Nephrology, Chicago, Illinois, ${ }^{2}$ Jesse Brown VA Medical \\ Center, Chicago, Illinois, ${ }^{3}$ Hektoen Institute of Medicine, Chicago, Illinois, ${ }^{4}$ Loyola University Medical Center, Maywood, Illinois.
}

Regenerative mechanisms after surgical injury have been studied in many organs but not in the kidney. Studying surgical injury may provide new insights into mechanisms of kidney regeneration. In rodent models, extrarenal tissues adhere to surgical kidney wound and interfere with healing. We hypothesized that this can be prevented by wrapping injured kidney in a plastic pouch. Adult rats tolerated 5/6 nephrectomy with pouch application well. Histological analysis demonstrates that application of the pouch effectively prevented formation of adhesions and induced characteristic wound healing manifested by formation of granulation tissue. Additionally, selected tubules of the wounded kidney extended into the granulation tissue forming branching tubular epithelial outgrowths (TEOs) without terminal differentiation. Tubular regeneration outside of renal parenchyma was not previously observed, and suggests previously unrecognized capacity for regeneration. Our model provides a novel approach to study kidney wound healing.

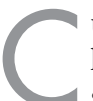
urrent efforts to regenerate functional kidney through the use of embryonic tissues and cells ${ }^{1-5}$ are hampered by ethical and safety concerns. While adult stem cells provide an alternative to embryonic sources, neither kidney-derived nor extra-renal adult stem cells have so far been shown to regenerate functional kidney tissue ${ }^{1-3,6-8}$. Thus, the challenge to find a viable novel approach to kidney regeneration remains open.

Wound healing after mechanical injury represents an important model for exploration of regenerative mechanisms in adult tissues ${ }^{9-16}$. It was demonstrated that surgical wounding of skin, muscle, and intestine leads to recruitment and expansion of adult progenitor cell pools ${ }^{13-15}$. Regeneration of the pancreas after pancreatectomy was shown to occur through the sequential dedifferentiation and redifferentiation of ductal epithelial cells ${ }^{16}$. While physiological recovery after surgical renal mass reduction has been extensively studied ${ }^{17-19}$, no attempts to study mechanisms of kidney healing at the wounded edge have been reported. There are two possible explanations for this lack. First, there is a prevalent belief that surgical resection of the adult mammalian kidney does not elicit regenerative responses ${ }^{7,20}$. Second, the surgical kidney injury in rodent models is technically complicated by the tendency of extrarenal tissues to adhere to the wound and interfere with the healing.

We hypothesized that extrarenal tissue adhesions can be prevented. Here we describe a model of surgical renal mass reduction in the adult rat kidney in which adhesions of the extrarenal tissues were prevented by enclosing polectomized kidney remnant into an inert plastic pouch ("pouch model"). Physiological data did not significantly differ between the control and experimental animals. We performed thorough comparative histological analysis of the wound healing with and without the interference of adhering tissues. At the wounded kidney edge, both in presence and in absence of the pouch, we observed tubular regeneration patterns similar to those previously described in classical models of acute tubular necrosis (ATN ${ }^{21-25}$. Additionally, in the kidney wound protected from adhesions by the pouch, we observed a novel pattern of tubular regeneration distinct from tubular regeneration described in ATN. In the pouch model, tubules of the injured kidney repaired outside of the kidney parenchyma in the surrounding granulation tissue and formed branching tubular epithelial structures lacking terminal differentiation. Our model provides a simple tool to study this previously unrecognized potential of adult mammalian kidney for regeneration.

\section{Results}

Wrapping kidney in plastic pouch effectively prevents extrarenal tissue adhesions without causing significant physiological changes. In control group of animals, in which polectomy was not followed by the pouch 
A

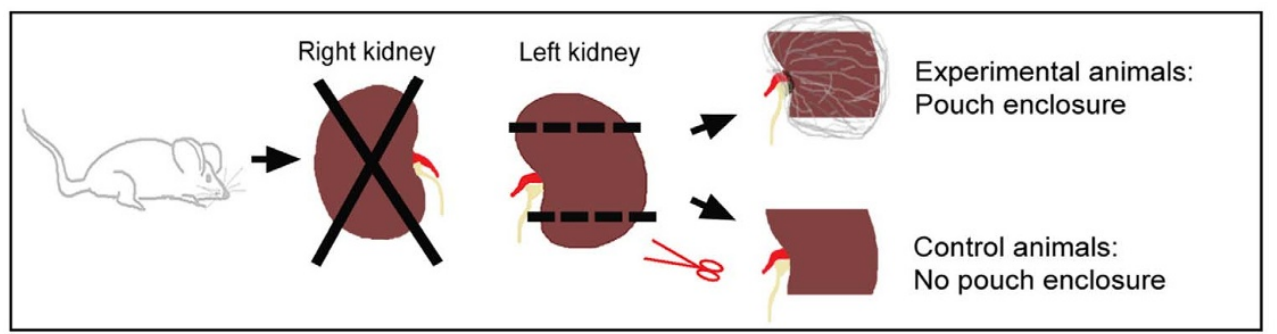

B

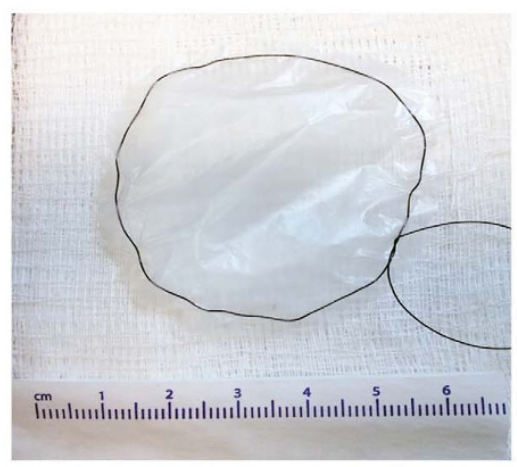

D

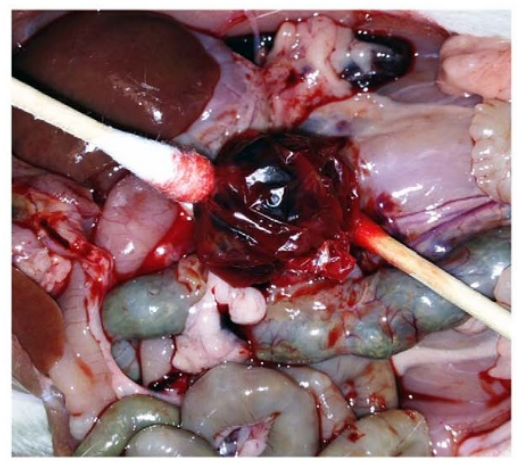

$\mathrm{F}$

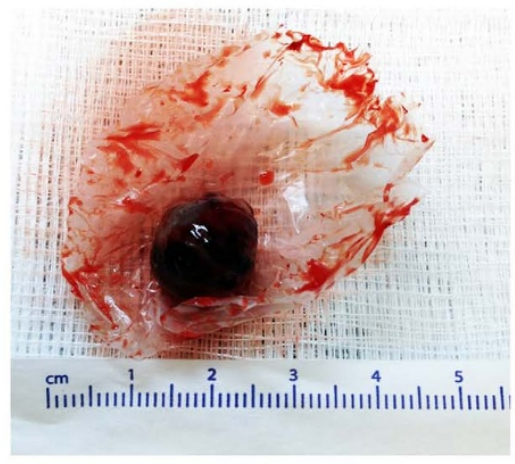

C

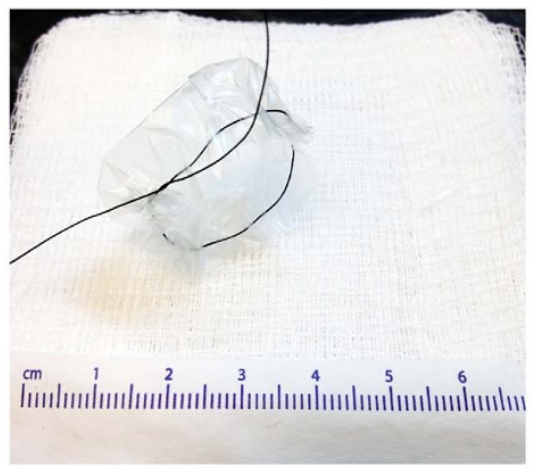

E

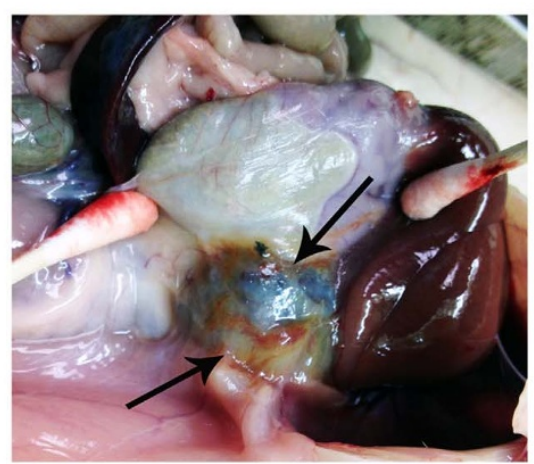

G

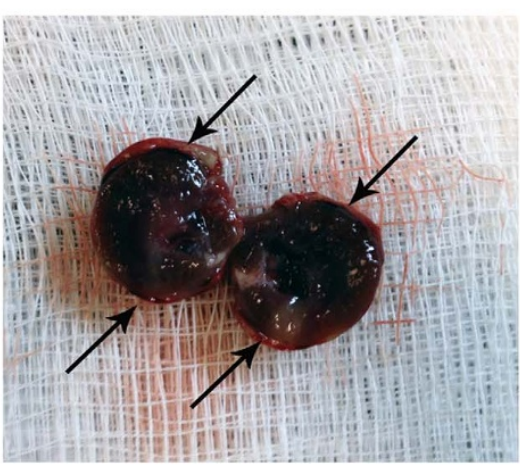

Figure $1 \mid$ A "pouch model" of 5/6 nephrectomy utilizes plastic pouch wrapping around the polectomized kidney. (A): Schematic representation of experimental design. (B): A plastic pouch device with relaxed sutures. (C): A plastic pouch device prepared for placement around the kidney by tightening sutures. (D): In vivo image of polectomized kidney wrapped in a pouch at two weeks after surgery demonstrates absence of extrarenal tissue adhesions. (E): In vivo image of the kidney in control animals without the pouch at two weeks after surgery demonstrates significant extrarenal tissue adhesions at both cut edges (arrows). (F): Kidney remnant extracted from experimental animal (pouch sutures removed). (G): Sagittal crossection of the kidney remnant extracted from experimental animal demonstrates absence of adhesions at the cut poles (arrows). Schematic drawing (A) by S.V. Photographs (BG) by N.L.

application (Fig. 1A, E), mostly fatty tissue adhesions (Fig. 1E, 2A \& C) but also pancreas and intestine adhesions (data not shown) were observed. In experimental animals in which polectomized kidney was wrapped in plastic pouch (Fig. 1A-D), adhesions of the extrarenal tissues were effectively prevented (Fig. 1D, F \& G). A distinct granulation tissue layer - defined as at least 10 cell-thick layer formed in the absence of adhesions - was overlying the wounded kidney edge (Fig. 2B \& D). These findings confirmed that extrarenal tissue adhesions were effectively prevented, and that a formation of distinct granulation tissue at the wound was 
A

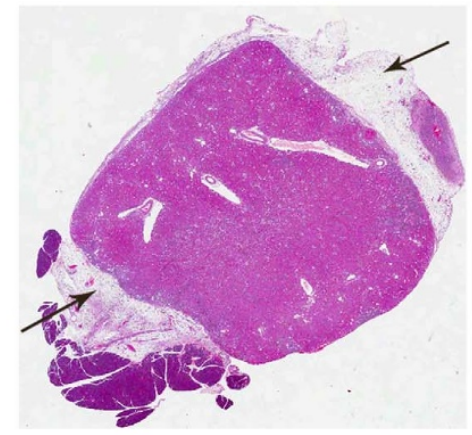

C

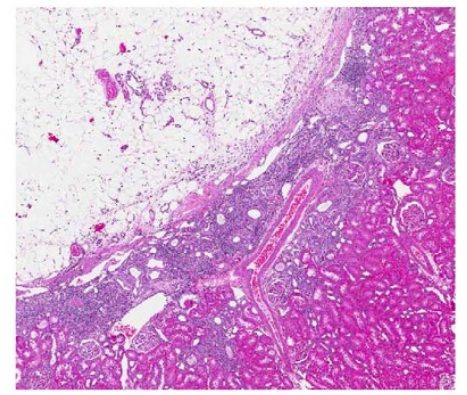

B

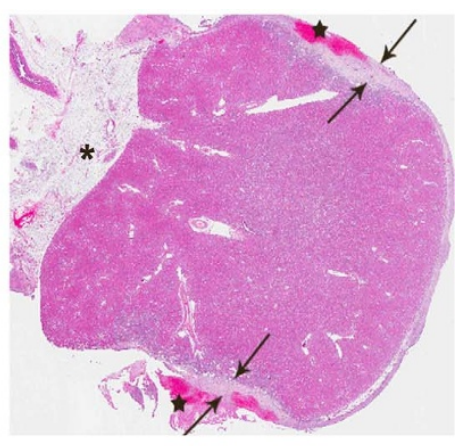

D

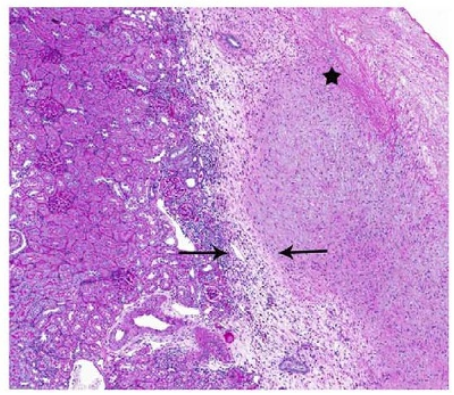

Figure $2 \mid$ Wrapping kidney in plastic pouch effectively prevents extrarenal tissue adhesions. (A): Low magnification view of the representative control kidney demonstrates extensive fatty tissue adhesions present at both cut edges of the polectomized kidney (arrows). H\&E, original magnification $3 x$. (B): Low magnification view of the representative experimental kidney demonstrates absence of adhesions and formation of a distinct granulation tissue layer at both cut edges of the polectomized kidney (arrows). Stars indicate blood clots. Asterisk indicates perirenal fat at the hilum. H\&E, original magnification 3x. (C): Higher magnification of the wounded kidney edge from a representative control animal demonstrates fatty tissue adhesions. H\&E, original magnification 40x. (D): Higher magnification of the wounded kidney from a representative experimental animal demonstrates absence of adhesions and formation of the granulation tissue layer (arrows). Star indicates blood clot overlying granulation tissue. PAS-hematoxylin. Original magnification $40 x$.

induced by enclosure of polectomized kidney within the inert plastic pouch.

Blood pressures, hemoglobin, blood urea nitrogen and serum creatinine levels were measured before and after surgery (Table 1). Rise in serum creatinine and blood urea was significant in both control and experimental groups as compared to preoperative measurements in naïve rats, but there was no significant difference between the control and experimental groups. There was no significant difference in blood pressures and hemoglobin measurements between animal groups. These data indicated that loose pouch application in 5/6 nephrectomy was well tolerated by the animals and did not produce Page kidney physiology.

Wounded kidney edge exhibits features of tubular regeneration. Kidney parenchyma at the cut edge displayed morphological features consistent with tissue remodeling both in the presence (Fig. 3A) and in the absence (Fig. 3B) of adhesions. Many tubules demonstrated morphological features of epithelial dedifferentiation: dense nuclei, increased nuclear-to-cytoplasmic volume ratio, decreased cytoplasmic eosinophilia, and a loss of brush border (Fig. 3C). Occasional apoptotic bodies and mitotic figures were present in tubular epithelium (data not shown). Some tubules appeared degenerated with flattened epithelium and dilated lumens. The interstitium of the wounded tissue contained variable amounts of inflammatory infiltrate and collagen deposits (Fig. 3D).

Additional histological findings confirmed tubular epithelial dedifferentiation at the wounded edge: diminished or lost expression of the terminal differentiation markers PHA (proximal tubule) ${ }^{26}$ (not shown), and Aqp2 (collecting duct $)^{27}$ (Fig. 3E), acquired expression of the mesenchymal marker vimentin (Fig. 3F), and re-expressed developmental markers $\mathrm{Pax}^{24}$ and $\mathrm{NCAM}^{23}$ (not shown). Quantification of these histological changes demonstrated that tubular dedifferentiation did not differ significantly between control and experimental tissues (Table 2).

Tubules of the pouch-wrapped injured kidney extend into the granulation tissue layer and form branching tubular epithelial structures. In experimental animal kidneys, where plastic pouch prevented adhesions, multiple tubular structures with predominantly cuboidal and columnar epithelium and thin PAS-positive basement membranes

Table 1 | Physiologic data obtained in animals preoperatively (Naive, $n=8)$, and two weeks after surgery in control $(C$ trl, $n=4)$ and experimental (Exper, $n=4)$ groups

\begin{tabular}{lccccc} 
Animals & $\mathrm{SBP}[\mathrm{mmHg}]$ & $\mathrm{DBP}[\mathrm{mmHg}]$ & $\mathrm{Hgb}[\mathrm{g} / \mathrm{dL}]$ & $\mathrm{BUN}[\mathrm{mg} / \mathrm{dL}]$ & $\mathrm{SCr}[\mathrm{mg} / \mathrm{dL}]$ \\
\hline Naive & $140 \pm 11$ & $83 \pm 8$ & $16.1 \pm 0.8$ & $19 \pm 4$ & $0.34 \pm 0.09$ \\
Controls & $142 \pm 10$ & $85 \pm 12$ & $14.6 \pm 1.2$ & $33 \pm 16$ & $39 \pm 11$ \\
Experimental & $157 \pm 20$ & $97 \pm 15$ & $14.6 \pm 1.0$ & $0.03 \pm 0.12$ & $0.75 \pm 0.15$ \\
P-value: Ctrl/Naive & 0.78 & 0.85 & 0.05 & 0.001 \\
P-value: Exper/Naive & 0.09 & 0.07 & 0.13 & 0.005 \\
P-value: Exper/Ctrl & 0.24 & 0.25 & 0.35 & 0.38 & 0.001 \\
\hline
\end{tabular}


A

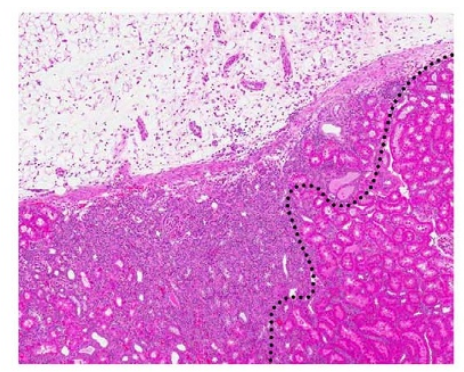

C

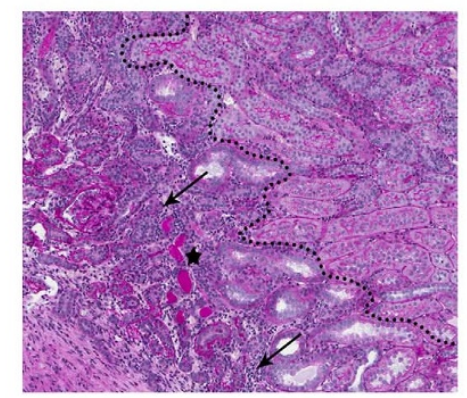

$\mathrm{E}$

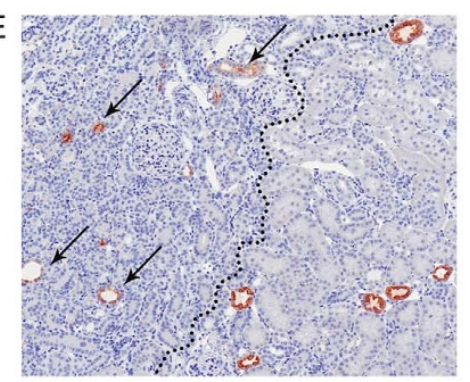

B

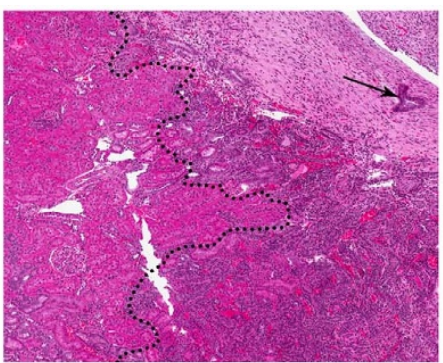

D

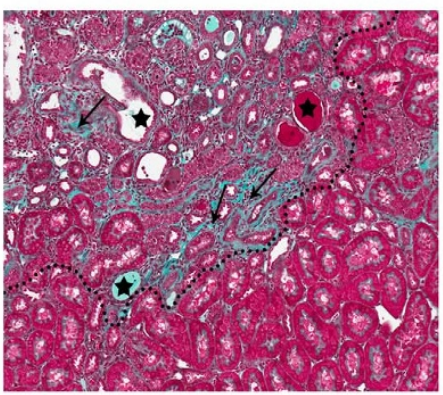

$\mathrm{F}$

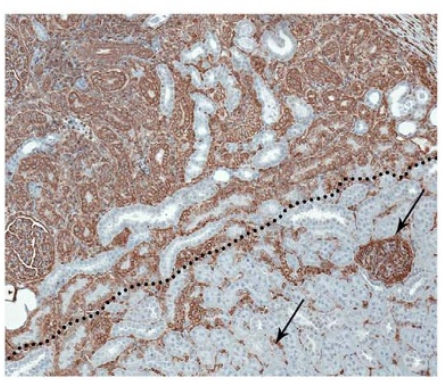

Figure 3 Wounded kidney edge exhibits features of tubular regeneration. (A), (B): Representative tissues from control (A) and experimental (B) animals demonstrate clear demarcation (hatched line) between morphologically preserved normal appearing renal parenchyma (A: right, $B$ : left) and altered renal parenchyma with features of remodeling and tubular dedifferentiation ( $A$ : left, $B$ : right). Fatty tissue adhesion is visible at the cut edge of control animal kidney (A: top). Thick granulation tissue layer is visible at the cut edge of experimental kidney (B: top-right). Tubular epithelial structure with columnar epithelium is visible within the granulation tissue (B: arrow). A, B: H\&E, original magnification 50x. (C), (D): Higher magnification of the cut edge demonstrates clear demarcation (hatched line) between morphologically preserved normal appearing renal parenchyma (right) and injured kidney parenchyma (left) with tubular epithelial cells dedifferentiation, increased nuclear-to-cytoplasm volume ratio, decreased cytoplasmic eosinophilia and lost brush border. Arrows indicate inflammatory infiltrates (C) and collagen deposits (D) in the interstitium. Stars indicate degenerated tubules with dilated lumens and flattened epithelium. C: PAS-hematoxylin, D: Gomori trichrome. (E): Immunostaining for Aqp2 - terminal differentiation marker of collecting ducts. Hatched line demonstrates clear demarcation between preserved renal parenchyma with normal expression (right) and injured parenchyma with diminished expression (left). Arrows indicate diminished or altered expression of Aqp2 in injured collecting ducts of the wounded kidney. (F): Immunostaining for mesenchymal marker vimentin. Hatched line demonstrates clear demarcation between preserved renal parenchyma with baseline expression (bottom) and injured parenchyma with increased expression (top). Arrows indicate normal vimentin distribution in glomeruli and intersitium. E, F: Hematoxylin counterstain. C-F: original magnification 100x.

were scattered throughout the granulation tissue (Fig. 3B \& 4A-C). The origin of tubular structures observed within the granulation tissue was explored by systematic tracing on serial sections. These structures proved to be connected to tubules of the wounded kidney (Fig. 4D-G, $\mathrm{H}-\mathrm{K})$.

In control animals neither distinct granulation tissue nor tubular epithelial structures outside the renal parenchyma were found on any of the serial sections studied (Fig. 2C \& 3A). This indicated that formation of adhesions interfered with the kidney wound healing, and prevented formation not only of granulation tissue layer but also of tubular structures within it.

Tubular structures within the granulation tissue are highly proliferative, strongly express epithelial markers and lack mesenchymal

Table 2 | Quantification of histological data. Areas of abnormal antigen expression were measured and expressed as percent of total section area ( $\mathrm{n}=4$ for each group)

\begin{tabular}{lcccccc} 
Histological stain & Gömori-trichrome & PHA & Aqp2 & Vimentin & Pax2 \\
\hline Controls & $7.0 \pm 2.7$ & $10.4 \pm 4.6$ & $12.2 \pm 5.5$ & $9.5 \pm 0.9$ & $5.4 \pm 1.6$ & $5.3 \pm 1.6$ \\
Experimental & $8.4 \pm 4.0$ & $7.0 \pm 1.1$ & $8.6 \pm 2.4$ & $8.4 \pm 2.6$ & $4.1 \pm 1.9$ & $4.0 \pm 2.9$ \\
P-value & 0.38 & 0.09 & 0.38 & 0.35 & 0.24 & 0.34 \\
\hline
\end{tabular}


A

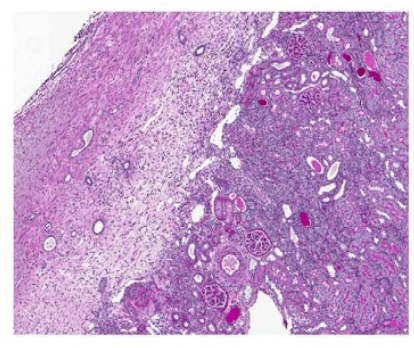

D

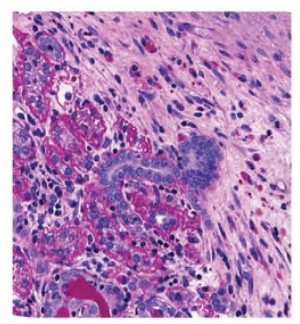

E
B
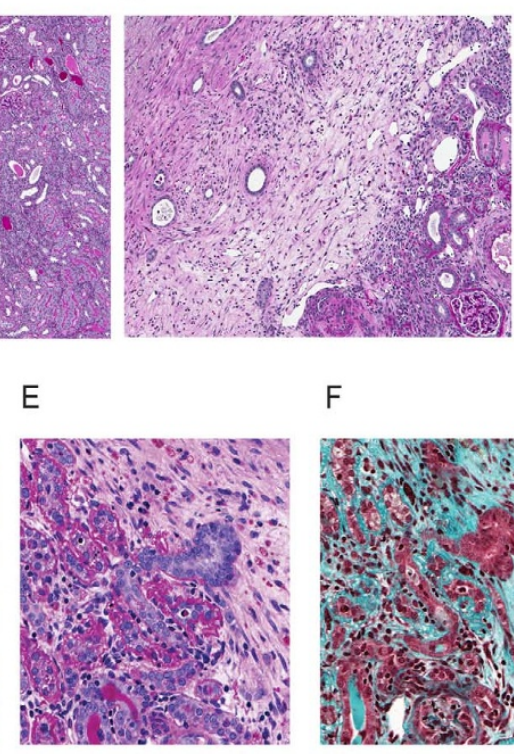

F

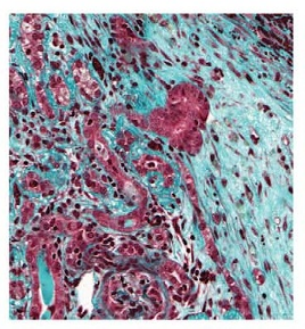

C

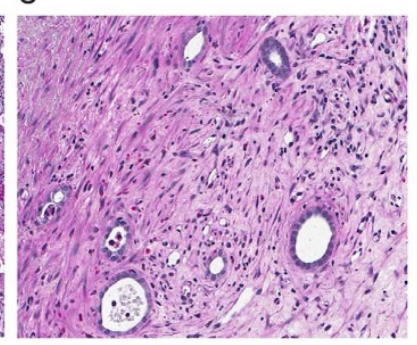

H

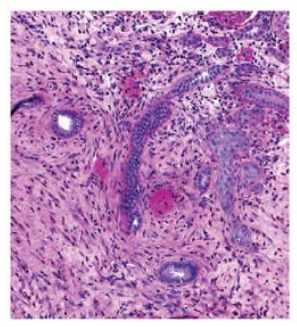

I

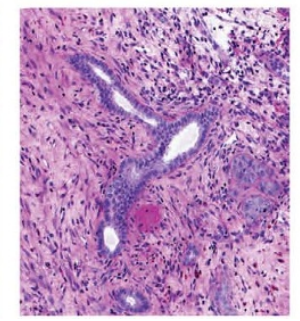

J

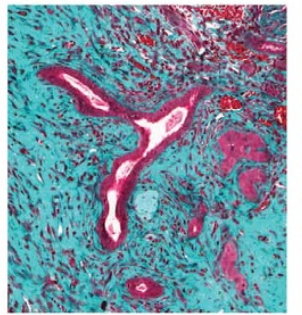

G

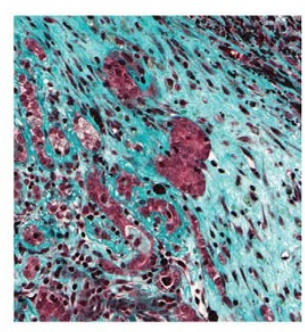

K

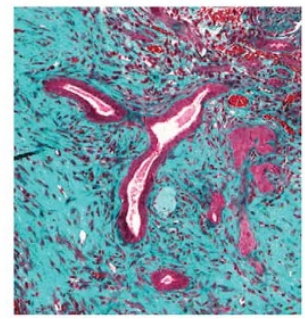

Figure $4 \mid$ Tubules of the pouch-wrapped injured kidney extend into the granulation tissue layer and form branching tubular epithelial structures. $(\mathrm{A}-\mathrm{C})$ : Representative tissue from experimental animal demonstrates several tubular epithelial structures formed within the granulation tissue layer. At a higher magnification these structures demonstrate cuboidal or columnar epithelium and a presence of thin PAS-positive basement membranes.

PAS-hematoxylin, original magnification A - 50x, B - 100x, C - 200x. (D-G) and (H-K): Serial sections of the wounded kidney from two experimental animals demonstrate tubular epithelial structures originating from tubules of the wounded tissue extending and branching within the granulation tissue layer. D, E and H, I: PAS-hematoxylin; F, G and J, K: Gömori trichrome stains. Original magnification 200x.

markers. Tubular epithelial structures outside the renal parenchyma could represent either remnant deteriorating tubules or proliferating regenerating tubules. To address this, we studied their morphology as well as expression of cell proliferation, and epithelial and mesenchymal differentiation markers. The following features of these structures suggested that they were regenerating rather than remnant tubules: (i) they had cuboidal or columnar epithelium (Fig. 4), consistent with healthy viable epithelium and markedly different from the flattened epithelium of deteriorating tubules; (ii) their epithelial cells were highly proliferative (Fig. 5A-C). Quantification of the three markers of cell proliferation (Fig. 5D) demonstrated that not only the percentage of proliferating cells in these tubules was 6- to 118-fold greater than that in normal tubular epithelium and collecting ducts (tubular structures within the granulation tissue layer vs. normal kidney tubules: PCNA $56.0 \pm$ $7.2 \%$ vs. $9.7 \pm 1.9 \%$; Ki67: $23.7 \pm 5.4 \%$ vs. $1.4 \pm 0.4 \%$; and $\mathrm{pHH} 3$ $11.9 \pm 3.6 \%$ vs. $0.1 \pm 0.1 \%$, with $p<0.05$ for all 3 comparisons), but that it was also 2- to 14 -fold greater than that in repairing tubules of the wounded kidney edge (tubular structures within the granulation tissue layer $v s$. wounded tubules: PCNA: $56.0 \pm 7.2 \% v$ s. $28.2 \pm 2.5 \%$, Ki67: $23.7 \pm 5.4 \%$ vs. $12.1 \pm 3.4 \%$, pHH3: $11.9 \pm 3.6 \%$ vs. $0.9 \pm$ $0.3 \%$; $p<0.05$ for all 3 comparisons); (iii) they strongly expressed epithelial differentiation markers panCK (Fig. 5E) and E-cadherin (Fig. 5F), typically lost or diminished in deteriorating tubules ${ }^{28,29}$; and (iv) they lacked expression of mesenchymal marker vimentin
(Fig. 5G) in contrast to strong vimentin expression expected in deteriorating tubular remnants ${ }^{28,30}$. Taken together these findings were consistent with tubular regeneration rather than degeneration, therefore the observed structures were named tubular epithelial outgrowths (TEOs).

TEOs exhibit irregular branching pattern distinct from normal collecting ducts. Dichotomous branching is infrequently encountered in collecting ducts of the normal adult kidney sections (Fig. 6AC). When followed on multiple serial sections, TEOs exhibited an irregular branching pattern (Fig. 6D-F) distinct from that expected in preexisting normal collecting ducts. This pattern of irregular branching in TEOs had two possible explanations: (i) they represented repairing preexisting collecting ducts with branching pattern distorted by the surrounding granulation tissue (pre-existing tubular regeneration), or (ii) they were formed by epithelial cells propagating over newly formed basement membranes (de novo tubular regeneration). Neither of these repair patterns has previously been described in adult mammalian kidney.

TEOs lack markers of proximal tubule, and express multiple markers of distal nephron. The next question we attempted to answer was whether TEOs where derived from proximal or distal epithelium of the nephron. TEOs lack of brush border and their capacity for branching (Fig. 4, Fig. 6D-F) indicated that they were more likely to 
A

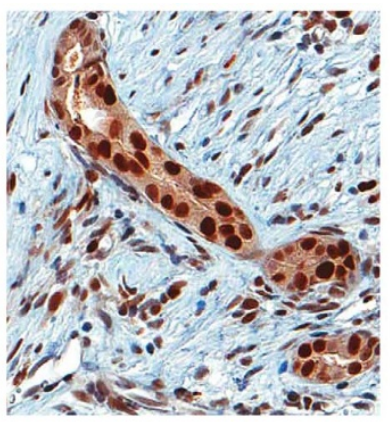

B

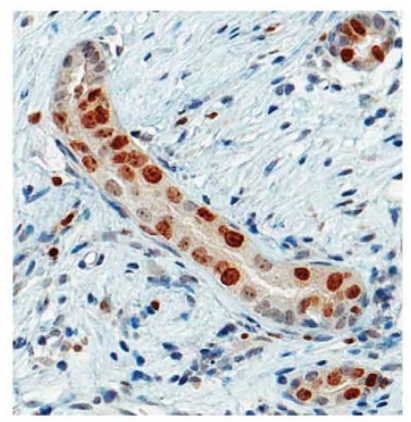

C

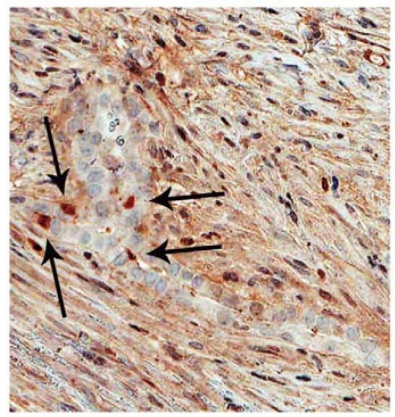

D

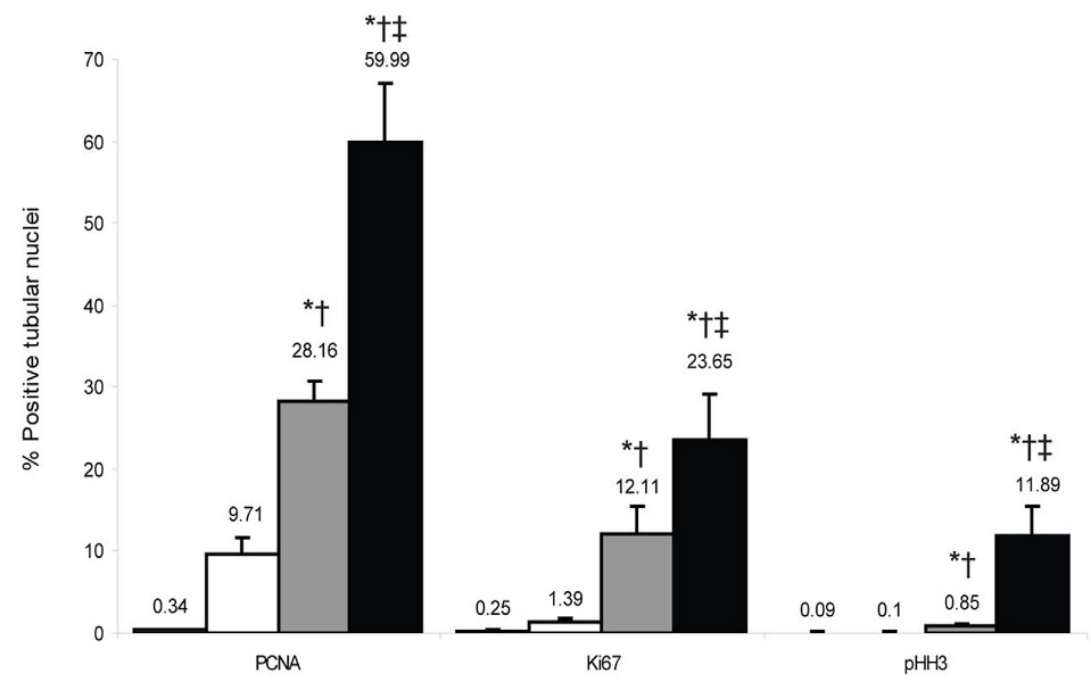

Proliferation marker

E

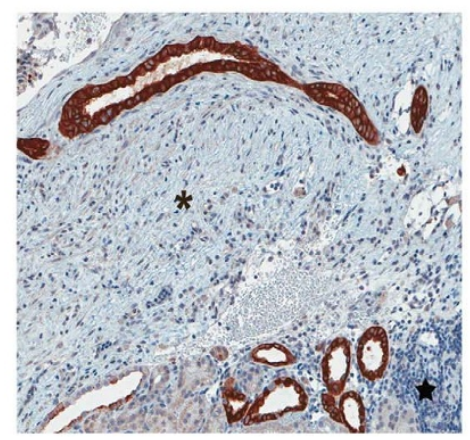

$\mathrm{F}$

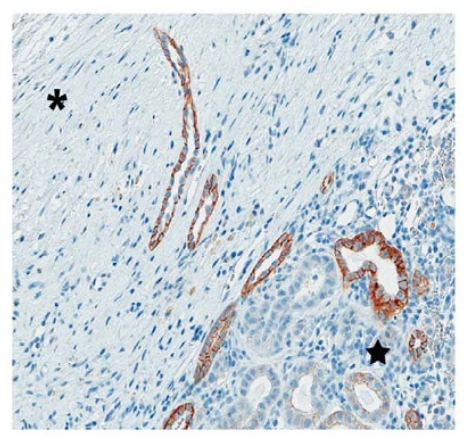

G

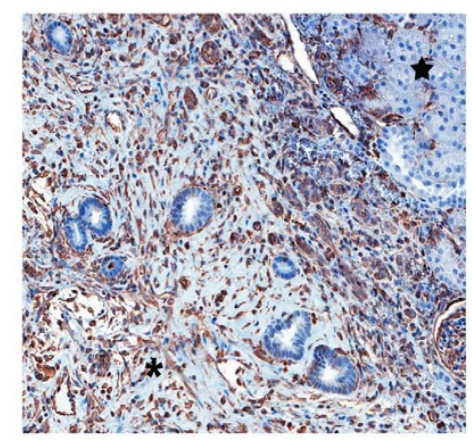

Figure 5 Tubular structures within the granulation tissue are highly proliferative, strongly express epithelial markers and lack mesenchymal markers. $(\mathrm{A}-\mathrm{C})$ : Immunostaining for cell proliferation markers demonstrates that tubular epithelial structures found within the granulation tissue layer of the wounded kidney are positive for PCNA (A), Ki67 (B), and mitotic marker pHH3 (arrows indicate positive nuclear stain in (C)). A-C: Hematoxylin counterstain; original magnification 200x. (D): Histogram demonstrates quantification of percent tubular epithelial cell nuclei positive for PCNA, Ki67 and $\mathrm{pHH} 3$ in collecting duct epithelium (crosshatched bars) and total tubular epithelium (white bars) of normal animals, in dedifferentiated epithelium of the wounded kidney of experimental animals (gray bars), and in tubular epithelial structures within the granulation tissue layer (black bars). Asterisks and daggers denote statistically significant difference as compared to normal tubules and collecting ducts respectively $(p<0.05)$. Double daggers denote statistically significant difference as compared to the tubules of wounded tissue $(p<0.05)$. (E-G): Immunostaining of the wounded tissue for epithelial markers panCK (E) and E-cadherin (F), and mesenchymal vimentin ( $\mathrm{G}$ ) demonstrates that tubular epithelial structures within the granulation tissue layer strongly express epithelial markers and lack expression of vimentin. Stars indicate wounded edge of the kidney. Asterisks indicate granulation tissue layer. E-G: Hematoxylin counterstain; original magnification 150x.

be derived from distal nephron. To confirm this, we performed immunostaining for proximal and distal tubular markers. Indeed, TEOs lacked PHA (proximal tubule terminal differentiation marker) (Fig. 7A), and were positive for several markers of collecting duct: $\mathrm{DBA}^{31}$ (Fig. 6E), Pax2 ${ }^{24}$ (Fig. 6F), calbindin-28kD ${ }^{32}$ (Fig. 7B), as well as PanCK $^{28}$ (Fig. 5E \& 6D) and E-cadherin ${ }^{33}$ (Fig. 5F). However, in contrast to normal distal nephron, TEOs mainly lacked expression of Aqp2 (Fig. 7C), a terminal differentiation marker of collecting 

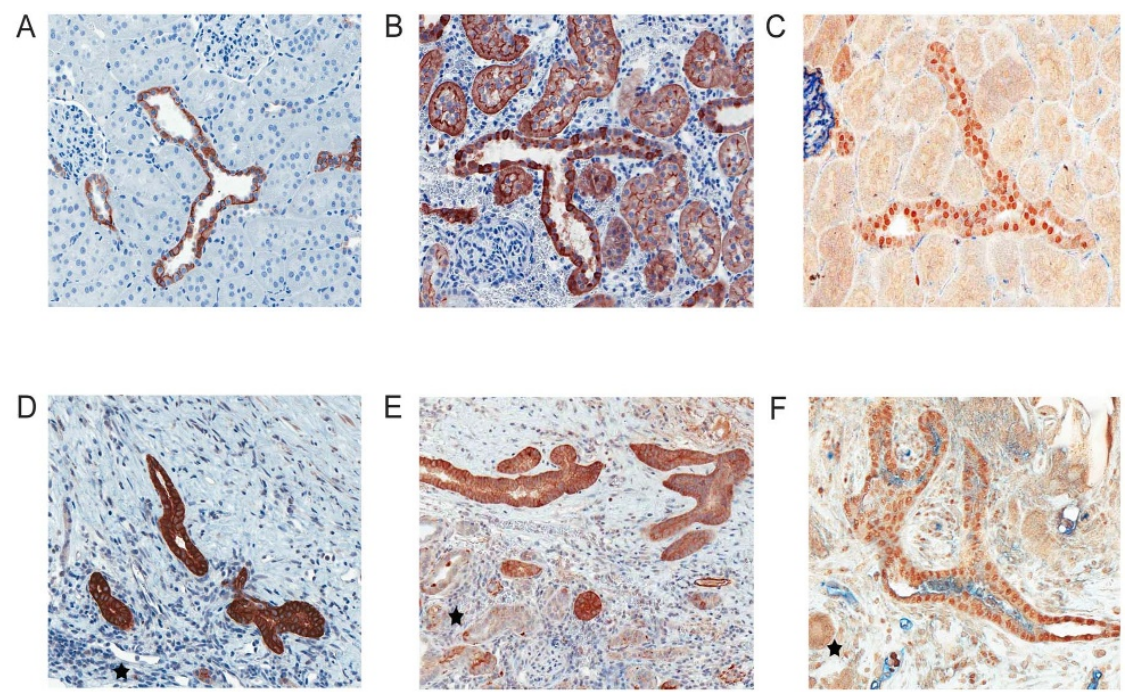

Figure 6 TEOs exhibit irregular branching pattern. (A-C): Regular dichotomous branching pattern of collecting ducts in normal kidney. (D-F): Irregular pattern of TEOs branching found within the granulation tissue layer. Stars indicate wounded kidney tissue. A, D: PanCK-hematoxylin, B, E: DBA-hematoxylin. C, F: Pax2-Podocalyxin. A-D \& F: original magnification 200x; E: original magnification 150x.

ducts $^{27}$. Diminished expression of Aqp2 was also observed in collecting ducts at the wounded edge of the kidney (Fig. 3E) indicating that TEOs likely originated from the injured collecting ducts of the wounded kidney.

\section{Discussion}

To explore the kidney's intrinsic capacity for regeneration, we developed a new model of surgical kidney injury in adult rat. The critical new feature of our model, as compared to previously studied models of surgical renal mass reduction ${ }^{17-19}$, was enclosure of the polectomized kidney in an inert plastic pouch ("pouch model"). This manipulation was introduced to prevent extrarenal tissues from adhering to the kidney wound and interfering with its healing. Physiological parameters such as blood pressure, hemoglobin, blood urea nitrogen and serum creatinine did not differ significantly between the experimental and control animal groups indicating that wrapping polectomized kidney in a loose-fitting pouch was well tolerated and did not induce Page kidney physiology. We also demonstrate that pouch enclosure effectively prevents extrarenal tissue adhesions to the kidney wound. A detailed histological analysis of the injured kidney at two weeks after surgery revealed features of remodeling and tubular dedifferentiation at the wounded edge. This was evident both in the presence of adhesions in controls, and in the absence of adhesions in experimental animal group. However, in contrast to controls, experimental animals demonstrated a distinct wound repair pattern manifested by formation of a thick granulation tissue layer overlying the wound. Additional remarkable feature of the pouch model was a presence of tubular extensions outside of the renal parenchyma in the granulation tissue layer. In control animals in the presence of adhesions, granulation tissue layer did not form, and no tubules extending beyond renal parenchyma were observed.

Tubules found within the granulation tissue layer were directly connected to tubules of the wounded kidney, and in theory could represent either degenerating tubular remnants or regenerating tubules. Their healthy cuboidal and columnar epithelium, marked expression of cell proliferation markers (PCNA, Ki-67, pHH3), as well as strong expression of epithelial (PanCK and E-cadherin) markers, and absence of mesenchymal marker vimentin were consistent with regeneration. We therefore named these structures tubular epithelial outgrowths (TEOs).
A

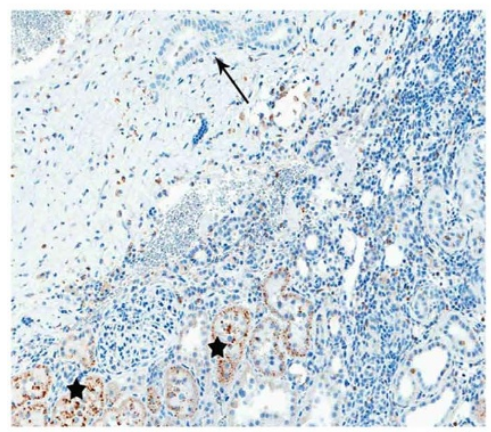

B

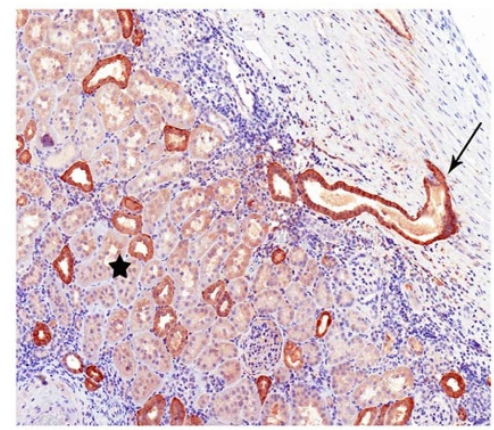

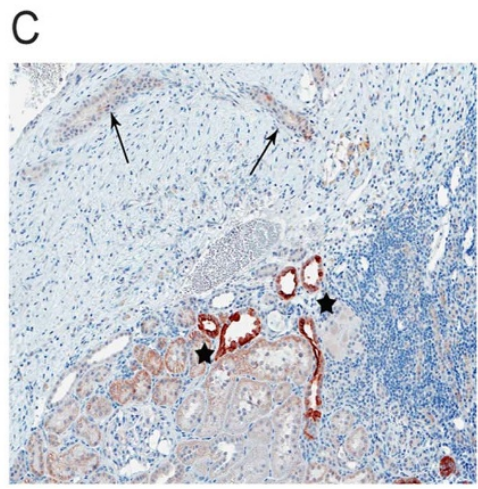

Figure 7 TEOs lack markers of proximal tubule and express multiple markers of distal nephron. (A): Immunostaining of the wounded kidney tissue for PHA demonstrates that TEOs lack expression of this proximal tubular marker. Arrow indicates PHA-negative TEO within the granulation tissue layer. Stars indicate proximal tubules within the wounded edge of the kidney with residual PHA stain. (B): Immunostaining of the wounded kidney tissue for clabindin-28kD demonstrates strong expression in TEOs (arrow). Star indicates clabindin-28kD positive collecting ducts within the wounded edge of the kidney. (C): Immunostaining of the wounded kidney tissue for Aqp2 indicates that TEOs lack expression of this collecting duct terminal differentiation marker. Arrows indicate TEOs within the granulation tissue. Stars indicate collecting ducts within the wounded tissue with residual Aqp2 expression. A-C: Hematoxylin counterstain; original magnification 100x. 
Regular dichotomous branching is present in collecting ducts of a normal adult kidney. TEOs displayed irregular branching pattern distinct from that of normal collecting ducts. Two potential mechanisms could account for formation of the irregularly branching TEOs. They were formed either by epithelial cells repopulating basement membrane of pre-existing collecting ducts trapped within the granulation tissue (repair of the pre-existing tubules), or by epithelial cells propagating over newly formed basement membranes outgrowing into the granulation tissue (de novo tubule regeneration). Both possibilities are of significant interest since they represent a unique pattern of tubular regeneration outside the kidney parenchyma previously not described in adult mammalian kidney in vivo. If TEOs represent true de novo tubular regeneration, this model can provide a new approach to the task of kidney regeneration. Equally remarkable is a possibility that TEOs represent repairing remnant collecting ducts. Remnant tubules devoid of proper parenchymal support are well known to undergo attrition and degeneration, therefore tubular repair within the granulation tissue indicates that a unique milieu promoting regeneration is created in the pouch model. Further studies are needed to determine precise mechanisms responsible for TEOs formation.

Renal tubular regeneration is best studied in classical models of tubular injury such as ischemia-reperfusion or nephrotoxic ATN $^{22-25}$. Many processes of tubular epithelial regeneration observed at the edge of the surgically wounded kidney were previously described in ATN: morphological dedifferentiation, loss of terminal differentiation markers, and re-expression of mesenchymal (vimentin) and developmental (Pax2 and NCAM) markers ${ }^{21-24}$. In ATN, dedifferentiated tubular epithelial cells propagate over pre-existing basement membranes and subsequently redifferentiate to acquire terminal differentiation and full functional capacity ${ }^{21,25}$. By contrast, tubular regeneration in our model led to formation of TEOs which lacked terminal differentiation, and therefore were unlikely to have functional capacity. Further studies are needed to determine whether TEOs have partial functional capacity.

Regardless of their function, TEOs formation is of great interest, because it indicates that adult renal tubules retain capacity to regenerate outside of renal parenchyma either by repair or by de novo tubulogenesis. The capacity of adult tubular epithelial cells to form new tubules is in agreement with previous data showing that adult tubules contain progenitor cells (reviewed in 8). Nonetheless, tubulogenesis has previously been described only in model systems using embryonic cells and immortalized kidney cell cultures ${ }^{34-38}$, but has not been reported in an adult mammalian kidney in vivo.

Absence of tubular regeneration outside of pre-existing renal parenchyma in ATN can be explained by minimal disruption of the surrounding parenchyma and tubular basement membranes. By contrast, in our model surgical kidney wounding allowed tubular repair outside of renal parenchyma. Also, significant disruption of the tubular basement membrane allowed dedifferentiated epithelial cells to migrate beyond the limits of the preexisting tubule. Although necessary, disruption of renal parenchyma and tubular basement membrane is not sufficient for TEOs formation as evident by the absence of TEOs in kidney wounds of control animals with adhesions. Pouch enclosure of the cut kidney prevented adhesions to the kidney wound, promoted formation of the granulation tissue layer, and created an enclosed environment that precluded diffusion of the cytokines and growth factors from the wound into surrounding tissue. Further studies are needed to delineate which of these factors and mechanisms promote formation of TEOs in the pouch model.

Epithelial cell capacity for dedifferentiation has been detected in both proximal ${ }^{22-25}$ and distal ${ }^{39-42}$ nephron epithelium. TEOs could thus be derived either from proximal or distal nephron. TEOs branching and lack of proximal tubular markers (brush border and PHA) suggested that they were derived from the distal nephron.
Accordingly TEOs expressed multiple markers of collecting duct epithelium (panCK, E-cadherin, DBA, Pax2, calbindin). However, TEOs expression of collecting ducts terminal differentiation marker Aqp2 was virtually absent. This was consistent with the loss of Aqp2 expression in the injured collecting ducts of the wounded kidney indicating dedifferentiation. Taken together, this data suggested that TEOs were derived from the injured collecting ducts undergoing dedifferentiation either by repair of the pre-existing collecting ducts or by de novo regeneration. Cell lineage studies are needed to confirm these findings.

In conclusion, we describe a novel "pouch model" of surgical kidney injury in adult rats. We demonstrate that enclosure of injured kidney within an inert plastic pouch effectively prevents adhesions of extrarenal tissues, and promotes a unique pattern of wound healing and tubular regeneration. Under the unique conditions created by pouch application, injured tubular epithelium regenerates beyond the pre-existing renal parenchyma to form branching tubular epithelial outgrowths (TEOs) without evident terminal differentiation. Our model provides a tool for studying the molecular and cellular mechanisms underlying this previously unrecognized regenerative potential of adult mammalian kidney.

\section{Methods}

All animal studies were performed according to the protocol approved by the Animal Care Committee and in accordance with the Animal Care Policies of the University of Illinois at Chicago.

Animals. Adult (10-12 weeks old) male Sprague-Dawley rats (Harlan, Indianapolis, IN) were used in accordance with IACUC standards and institutionally approved protocols.

Surgical model. In experimental animals following right nephrectomy, the left renal artery was reversibly ligated. The upper and lower poles of the left kidney were resected using surgical scissors. Approximately 5/6 renal mass reduction was achieved. Sterile gauze was applied to the cut surface for hemostasis for 1-2 minutes. The remnant kidney was enclosed in a sterile in-house-made virgin high-density polyethylene (HDPE) pouch (Fig. 1A-D) that was loosely fastened around the kidney's pelvis. The left renal artery ligature was then removed (total left kidney ischemia time 3-5 minutes), and the laparotomy site was closed. The HDPE was selected as a pouch material because it is impermeable, non-leaching, biologically inert and amenable to sterilization. Control animals underwent the identical procedure (including renal artery ligation for 3-5 minutes) with the exception that the pouch was not applied (Fig. 1A). Post-operatively all rats received $10 \mathrm{~mL}$ of lactated ringer solution, Ampicillin (Novaplus ${ }^{\circledR}[250 \mathrm{mg} / \mathrm{mL}]$ Sandoz, Austria), and buprenorphine (Buprenex ${ }^{\circledR}[0.3 \mathrm{mg} / \mathrm{mL}]$ Reckitt Benckiser, UK). Standard rat chow was given ad libitum. Animals were sacrificed at two weeks post-operatively. At least four animals were studied in each of experimental and control groups. Nephrectomized kidneys were used as normal adult kidney controls for immunostaining.

Physiological data. Blood pressures were measured by the tail cuff method ${ }^{43}$ on previously trained and habituated animals. Whole blood hemoglobin was measured using HemoCue ${ }^{\circledR} \mathrm{Hb} 201^{+}$(Angelholm, Sweden) analyzer. Blood urea nitrogen and serum creatinine analyses were done by the Biological Resource Laboratory of the University of Illinois at Chicago.

Histology and immunostaining. When tissues were harvested, pouch was removed if present, and kidney remnant was removed from the pouch (Fig. 1F), and cut sagittally into two halves (Fig. 1G). Tissue was then embedded and cut serially. 200300 sections at $5 \mu \mathrm{m}$ thickness for each kidney half were studied. Cut sections were placed on histological slides, deparafinized and subjected either to routine hematoxylin and eosin (H\&E), periodic acid Schiff-hematoxylin (PAS-H), and Gömori trichrome stains or to the immunostaining. For specific antigen stains antigen retrieval was performed followed by blocking and sequential incubation with primary and secondary antibodies. Primary antibodies used were: anti-aquaporin 2 (Aqp2) (NB110-74682, Novus Biologicals, Littleton, CO); anti-calbindin (28kD) (ab11426, Abcam, Cambridge, MA); anti-Dolichos biflorus agglutinin (DBA) (L6533, Sigma, St. Louis, MO); anti-E-cadherin (18-0223, Invitrogen, Camarillo, CA); antiKi67 (M7248, Dako, Carpinteria, CA); anti-neural cell adhesion molecule (NCAM) (5B8, DSHB, Iowa City, IA); anti-Pax2 (PRB-276-P, Covance, Emeryville, CA); antipan-cytokeratin (panCK) (C1801, Sigma, St. Louis, MO); anti-podocalyxin (AF1556, R\&D Systems, USA); anti-proliferating cell nuclear antigen (PCNA) (MAB424, Millipore, USA); anti-Phaseolus vulgaris agglutinin (PHA) (BA 0224, Vector, Burlingame, CA); anti-phosphorylated-histone H3 (pHH3) (H0412, Sigma, St. Louis, MO); anti-vimentin (V6389, Sigma, St. Louis, MO). Elite ABC Reagent and Alkaline Phosphatase Avidine D were used, and reactions developed with 3, 
$3^{\prime}$-diaminobenzidine (DAB) and/or Alkaline Phosphatase (AP) Substrate kit III (Vector, Burlingame, CA). Single stains were counterstained with hematoxylin.

Whole-slide digital microscopy, quantification, and statistical analysis. Whole slide digital image acquisition was performed using the Aperio ScanScope ${ }^{\circledR}$ CS System (Aperio Technologies, Vista, CA) at $200 \times$ magnification. Four sections per animal were randomly selected from slides stained for Gömori trichrome and each specific marker from control and experimental groups. An independent investigator quantified areas of abnormal antigen expression where abnormal antigen expression was defined as an immunostaining pattern different from the normal adult rat kidney immunostaining pattern. Total and abnormal section areas were marked and measured by the Aperio software pen tool. The final results were expressed as a percent of total section area, and the acquired percentage means were compared by Student's t-test (Table 2).

Nuclear PCNA, Ki67, pHH3 were quantified in tissues using Aperio Nuclear v9 algorithm, version 9.1. Two representative fields from four different animals were annotated using the software for the cortex of normal kidneys, and the wounded tissue and TEOs of experimental kidneys. Total tubular and collecting duct nuclei were counted, and the percentage of positive nuclei was determined within each field. The means of percentages were compared by Student's t-test.

1. Little, M. Regrow or repair: potential regenerative therapies for the kidney. J. Am. Soc. Nephrol. 17, 2390-2401 (2006).

2. Yokoo, T., Matsumoto, K. \& Yokote, S. Potential use of stem cells for kidney regeneration. Int. J. Nephrol. 2011, 1-9 ID591731 (2011).

3. Litbarg, N. O., Vujicic, S. \& Singh, A. K. Attempts to Regenerate the Mammalian Kidney. In: Ing, T. S., Rahman, M. \& Kjellstrand, C. M. editors. Dialysis: History, development and promise. London: Imperial College Press, Chapter 8. (2012).

4. Xinaris, C. et al. In vivo maturation of functional renal organoids formed from embryonic cell suspensions. J. Am. Soc. Nephrol. 23, 1857-1868 (2012).

5. Mae, S.-I. et al. Monitoring and robust induction of nephrogenic intermediate mesoderm from human pleuripotent stem cells. Nat. Commun. 4, 1367 (2013)

6. Benigni, A., Morigi, M. \& Remuzzi, G. Kidney regeneration. Lancet 375, 1310-1317 (2010)

7. Anglani, F. et al. The regenerative potential of the kidney: what can we learn from developmental biology. Stem. Cell Rev. 6, 650-657 (2010).

8. Romagnani, P., Lasagni, L. \& Remuzzi, G. Renal progenitors: an evolutionary conserved strategy for kidney regeneration. Nat. Rev. Nephrol. 9, 137-146 (2013).

9. Prisk, V. \& Huard, J. Muscle injuries and repair: the role of prostaglandins and inflammation. Histol. Histopathol. 18, 1243-1256 (2003).

10. Navarro, X., Vivo, M. \& Valero-Cabre, A. Neural plasticity after peripheral nerve injury and regeneration. Prog. Neurobiol. 82, 163-201 (2007).

11. Yu, B. D., Mukhopadhyay, A. \& Wong, C. Skin and hair: models for exploring organ regeneration. Hum. Mol. Genet. 17, R54-R59 (2008).

12. Stappenbeck, T. \& Miyoshi, H. The role of stromal stem cells in tissue regeneration and wound repair. Science 324, 1666-1669 (2009).

13. Levy, V., Lindon, C., Zheng, Y., Harfe, B. D. \& Morgan, B. A. Epidermal stem cells arise from the hair follicle after wounding. FASEB J 21, 1358-1366 (2007).

14. Nesti, L. J. et al. Differentiation potential of multipotent progenitor cells derived from war-traumatized muscle tissue. J. Bone Joint Surg. Am. 90, 2390-2398 (2008)

15. Dekaney, C. M. et al. Expansion of intestinal stem cells associated with long-term adaptation following ileocecal resection in mice. Am. J. Physiol. Gastrointest. Liver Physiol. 293, G1013-G1022 (2007).

16. Li, W. C. et al. Activation of pancreatic-duct-derived progenitor cells during pancreas regeneration in adult rats. J. Cell Sci. 123, 2792-2802 (2010).

17. Griffin, K. A., Picken, M. \& Bidani, A. K. Method of renal mass reduction is a critical modulator of subsequent hypertension and glomerular injury. J. Am. Soc. Nephrol. 4, 2023-2031 (1994).

18. Ibrahim, H. N. \& Hostetter, T. H. The renin-aldosterone axis in two models of reduced renal mass in the rat. J. Am. Soc. Nephrol. 9, 72-76 (1998).

19. Kang, D.-H., Hughes, J., Mazzali, M., Schreiner, G. F. \& Johnson, R. J. Impaired angiogenesis in the remnant kidney model. II. Vascular endothelial growth factor administration reduces renal fibrosis and stabilizes renal function. J. Am. Soc. Nephrol. 12, 1448-1457 (2001).

20. Little, M. H. Tracing the life of the kidney tubule - re-establishing dogma and redirecting the options. Cell Stem Cell 2, 191-192 (2008).

21. Bacallao, R. \& Fine, L. G. Molecular events in the organization of renal tubular epithelium: From nephrogenesis to regeneration. Am. J. Physiol. 257, F913-F924 (1989).

22. Witzgall, R., Brown, D., Schwarz, C. \& Bonventre, J. V. Localization of proliferating cell nuclear antigen, vimentin, c-Fos, anclusterin in the postischemic kidney. Evidence for a heterogenous genetic response among nephron segments, and a large pool of mitotically active and dedifferentiated cells. J. Clin. Invest. 93, 2175-2188 (1994)

23. Abbate, M., Brown, D. \& Bonventre, J. V. Expression of NCAM recapitulates tubulogenic development in kidneys recovering from acute ischemia. Am. J. Physiol. 277(3 Pt 2), F454-F463 (1999).
24. Imgrund, M. et al. Re-expression of the developmental gene Pax-2 during experimental acute tubular necrosis in mice 1. Kindey Intl. 56, 1423-1431 (1999)

25. Bonventre, J. Dedifferentiation and proliferation of surviving epithelial cells in acute renal failure. J. Am. Soc. Nephrol. 14, S55-S61 (2003).

26. Khan, T. N. \& Sinniah, R. Study of renal tubular glycoconjugates in tubulointerstitial damage using conjugated lectins. J. Pathol. 170, 187-196 (1993).

27. Yamamoto, T. et al. Expression of AQP family in rat kidneys during development and maturation. Am. J. Physiol. 272 (2 Pt 2), F198-204 (1997).

28. Moll, R., Hage, C. \& Thoenes, W. Expression of intermediate filament proteins in fetal and adult human kidney: modulations of intermediate filament patterns during development and in damaged tissue. Lab. Invest. 65, 74-86 (1991).

29. Vongwiwatana, A., Tasanarong, A., Rayner, D. C., Melk, A. \& Halloran, P. F. Epithelial to mesenchymal transitioning during late deterioration of human kidney transplants: the role of tubular cells in fibrogenesis. Am. J. Transpl. 5, 1367-1374 (2005).

30. Kers, J. et al. Intragraft tubular vimentin and CD44 expression correlate with longterm renal allograft function and interstitial fibrosis and tubular atrophy. Transplantation 90, 502-509 (2010).

31. Holthofer, H., Schulte, B. A. \& Spicer, S. S. Expression of binding sites for Dolichos biflorus agglutinin at the apical aspect of collecting duct cells in rat kidney. Cell Tissue Res. 249, 481-485 (1987).

32. Taylor, A. N., McIntosh, J. E. \& Bordeau, J. E. Immunocytochemical localization of vitamin $\mathrm{D}$-dependent calcium-binding protein in renal tubules of rabbit, rat and chick. Kidney Int. 21, 765-773 (1982).

33. Prozialeck, W., Lamar, P. C. \& Appelt, D. M. Differential expression of Ecadherin, N-cadherin and beta-catenin in proximal and distal segments of the rat nephron. BMC Physiol. 4, 10 (2004).

34. Montesano, R., Schaller, G. \& Orci, L. Induction of epithelial tubular morphogenesis in vitro by fibroblast-derived soluble factors. Cell 66, 697-711 (1991).

35. Sakurai, H., Barros, E. J., Tsukamoto, T., Barasch, J. \& Nigam, S. K. An in vitro tubulogenesis system using cell lines derived from the embryonic kidney shows dependence on multiple soluble growth factors. Proc. Natl. Acad. Sci. U S A 94, 6279-6284 (1997).

36. Kobayashi, T. et al. Wnt4-transformed mouse embryonic stem cells differentiate into renal tubular cells. Biochem. Biophys. Res. Commun. 336, 585-595 (2005).

37. Yamamoto, M. et al. Branching ducts similar to mesonephric ducts or ureteric buds in teratomas originating from mouse embryonic stem cells. Am. J. Physiol. Renal Physiol. 290, F52-F56 (2006)

38. Kelly, K. J., Kluve-Beckerman, B. \& Dominguez, J. H. Acute-phase response protein serum amyloid A stimulates renal tubule formation: studies in vitro and in vivo. Am. J. Physiol. Renal Physiol. 296, F1355-F1363 (2009).

39. Baer, P. C., Tunn, U. W., Nunez, G., Scherberich, J. E. \& Geiger, H. Transdifferentiation of distal but not proximal tubular epithelial cells from human kidney in culture. Exp. Nephrol. 7, 306-313 (1999).

40. Sun, D. F. et al. Relation of distal nephron changes to proximal tubular damage in uranyl acetate-induced acute renal failure in rats. Am. J. Nephrol. 22, 405-416 (2002).

41. Ivanova, L., Butt, M. J. \& Matsell, D. G. Mesenchymal transition in kidney collecting duct epithelial cells. Am. J. Physiol. Renal. Physiol. 294, F1238-F1248 (2008).

42. Aldehni, F. et al. Bestrophin 1 promotes epithelial-to-mesenchymal transition of renal collecting duct cells. J. Am. Soc. Nephrol. 20, 1556-1564 (2009).

43. Kubota, Y. et al. Evaluation of blood pressure measured by tail-cuff methods (without heating) in spontaneously hypertensive rats. Biol. Pharm. Bull. 29(8), 1756-1758 (2006).

\section{Acknowledgements}

The authors wish to thank Matthew Lindeblad for his valuable comments and technical assistance with the experimental part of this work.

\section{Author contributions}

All authors reviewed the manuscript. N.L. and S.V. designed experiments, performed research, analyzed the data, and wrote the paper. P.S. and S.S. performed experiments. S.S., G.D., J.A. and A.S. analyzed the data, and wrote the paper.

\section{Additional information}

Competing financial interests: The authors declare no competing financial interests.

How to cite this article: Litbarg, N.O. et al. A Novel Model of Surgical Injury in Adult Rat Kidney: A “Pouch Model”. Sci. Rep. 3, 2890; DOI:10.1038/srep02890 (2013).

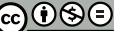

This work is licensed under a Creative Commons AttributionNonCommercial-NoDerivs 3.0 Unported license. To view a copy of this license, visit http://creativecommons.org/licenses/by-nc-nd/3.0 
SUBJECT AREAS: DIFFERENTIATION ANIMAL DISEASE MODELS KIDNEY CELL PROLIFERATION SCIENTIFIC REPORTS: $3: 2890$ DOI: $10.1038 /$ srep02890 (2013)

Published: 8 October 2013 Updated: 13 January 2014
ERRATUM: A Novel Model of Surgical Injury in Adult Rat Kidney: A "Pouch Model" $^{\prime \prime}$

Natalia O. Litbarg, Snezana Vujicic, Suman Setty, Periannan Sethupathi, George Dunea, Jose A. Arruda \& Ashok K. Singh

A file entitled 'Supplementary Info' was inadvertently published as Supplementary Information with this Article. This file has now been removed. 